\title{
Prognostic Factors and Survival Outcome of Whole Brain Radiotherapy in Metastatic Brain Cancer- A Single Regional Cancer Centre Experience in North India
}

\author{
Purnima Thakur¹, Aman Sharma², Manish Gupta ${ }^{3}$, Anupama Dhiman4, Jyoti Sharma ${ }^{5}$
}

${ }^{1}$ Department of Radiotherapy, Indira Gandhi Medical College, Shimla, Himachal Pradesh, India. ${ }^{2}$ Department of Radiotherapy, Indira Gandhi Medical College, Shimla, Himachal Pradesh, India. ${ }^{3}$ Department of Radiotherapy, Indira

Gandhi Medical College, Shimla, Himachal Pradesh, India. ${ }^{4}$ Department of Community Medicine, Sri Lal Bahadur Shastri Government Medical College, Mandi, Himachal Pradesh, India. ${ }^{5}$ Department of Radiotherapy, Indira Gandhi Medical College, Shimla, Himachal Pradesh, India.

\section{ABSTRACT}

\section{BACKGROUND}

Brain metastasis is the leading cause of mortality and morbidity and is the most common intracranial neoplasm in adults. Once the brain metastasis develops, treatment remains only palliative with an intention to improve the quality of life. Whole brain radiotherapy is the palliative treatment offered to such patients at our institute. This study is the first of its kind in Northern India which aims to determine the survival after whole brain radiotherapy and prognostic factors of brain metastasis from solid tumors.

\section{METHODS}

This is a retrospective study conducted at a tertiary cancer centre in Northern India treated over a period of 5 years from 2014 to 2018. The data was collected from the hospital database and details of treatment and patient characteristics were collected from patient's treatment charts. The data was entered into Microsoft Office Excel 2007 and analysed with the help of SPSS Ver. 20.

\section{RESULTS}

Median survival was found to be 3.7 months ( 0.03 to 21.9 months). On univariate analysis, median survival was found to be significantly higher among patients with less than three brain mets ( $p=0.003)$, size of brain mets less than $3 \mathrm{cms}(\mathrm{p}=0.005)$ and dose of radiotherapy more than or equal to $30 \mathrm{~Gy}(\mathrm{p}=0.001)$. On multivariate analysis also, the number of BM, size of BM and dose of radiotherapy were found to be significant predictors of overall survival.

\section{CONCLUSIONS}

Though whole brain radiotherapy improves survival in such patients, the benefit offered is small. Based on prognostic factors, we can identify such group of patients who may just be offered best supportive care. This may decrease the palliative workload in a limited resource setting like ours. This result must be interpreted with caution since the administration of steroids was not controlled in the present study.
Corresponding Author: Dr. Purnima Thakur, Department of Radiotherapy, Indira Gandhi Medical College, Shimla, Himachal Pradesh, India.

E-mail: purnimathakur28@gmail.com

DOI: $10.14260 /$ jemds/2019/695

Financial or Other Competing Interests: None.

How to Cite This Article:

Thakur P, Sharma A, Gupta $M$, et al. Prognostic factors and survival outcome of whole brain radiotherapy in metastatic brain cancer- a single regional cancer centre experience in North India. J. Evolution Med. Dent. Sci. 2019;8(43):32063211, DOI: 10.14260/jemds/2019/695

Submission 18-08-2019, Peer Review 10-10-2019, Acceptance 18-10-2019, Published 28-10-2019.

\section{KEY WORDS}

Brain Metastasis, Palliation, Radiotherapy, Prognostic Factors 


\section{BACKGROUND}

Brain is a common metastatic site in many malignancies, most common being lung cancer, followed by cancer breast and then gastrointestinal sites. 20 to $40 \%$ of cancer patients are estimated to develop brain metastasis during the course of their illness.[1] $30-50 \%$ patients of NSCLC, $10-20 \%$ of all and $38 \%$ of HER 2 neu positive metastatic breast cancer experience brain metastasis. [2,3,4] With the increasing use of chemotherapy and advancement in radiotherapy techniques, life expectancy of cancer patients is increasing and consequently, more patients are presenting with brain secondaries.

Brain metastasis is itself a poor prognostic factor in malignancy as it limits the survival and worsens the quality of life in cancer patients. Median survival of 1-2 months has been estimated without any treatment. $[5,6]$

The treatment modalities used for brain metastases are corticosteroids to decrease peritumoural oedema, antiepileptic drugs for treatment of seizures, chemotherapy, whole brain radiotherapy (WBRT), radiosurgery and surgery if indicated may be used alone or in combination. [7] Multidisciplinary approach integrating surgical, radiation, and chemotherapeutic options may benefit the patient.

WBRT is a palliative intervention in the treatment of brain metastasis and plays role in improvement of neurological complaints with minimal morbidity. There is a small subset of patients with single or oligometastatic brain metastasis who can be treated by surgical or targeted radiotherapy approaches such as stereotactic radiosurgery/radiotherapy (SRS or SRT) with or without WBRT with an intention to treat, while in rest of the patients WBRT has a palliative intent. Nonrandomized studies suggest that WBRT increases median survival up to three to six months, although extra cranial disease may dictate the ultimate outcome in many patients. [8]

Determination of pre-treatment prognostic factors becomes important in these patients, as it may guide in proper selection of patients of secondary brain who may benefit from WBRT. For patients with poor performance status, best supportive care may be the best treatment offered.

This retrospective study was conducted on the patients with brain metastases from solid tumors, treated at our institution in a 5-year period. The aim of this study is to determine median survival and overall survival in these patients of BM (brain metastasis) after receiving WBRT and to identify independent prognostic pretreatment factors in patients with brain metastases. We analyzed several parameters including age, primary site, control of primary tumor, number of brain metastasis, size of metastatic tumor and total dose of radiotherapy received. We also performed a review of the literature, on the prognostic factors and the results were compared with findings from our database.

\section{METHODS}

The study was conducted at a tertiary cancer centre in Northern India which provides comprehensive treatment and care to cancer patients. The study sample included database of patients treated at Department of Radiotherapy and Oncology between 2014 and 2018. Ninety-seven patients were included in the study as per inclusion and exclusion criteria.

\section{Methods of Data Collection}

Data was collected by author after reviewing patient's charts. From the database, all patients who were diagnosed with brain mets that had spread from a primary site outside brain, during study period were identified. The treatment modalities of these patients were reviewed from patient's charts. Data regarding demographics, symptoms, characteristics of brain mets, course of illness and survival were also collected.

\section{Inclusion and Exclusion Criteria}

Only those patients who were diagnosed with brain mets and treated with WBRT alone or along with adjuvant chemotherapy were included in this study. Patients with incomplete medical records, including details of WBRT administration, were excluded. Patients undergoing any kind of surgical treatment (Biopsy or resection) and those treated with stereotactic radiosurgery (SRS) were also excluded.

\section{Assessment of Brain mets and Symptom Relief}

$\mathrm{BM}$ was assessed and measured using contrast-enhanced MRI or CT for the initial assessment. Follow up scan were not included in the assessment. As the follow up scans of very few patients was available, local control could not be assessed accurately. The palliative effect of WBRT was difficult to assess in patients due to prior administration of dexamethasone. As a proxy measure for symptom relief provided by WBRT, Karnofsky performance status (KPS) of patients was determined.

\section{Statistical Analysis}

The data was entered into Microsoft Office Excel 2007 and analysed with the help of SPSS 20v. Descriptive statistical analysis was represented through frequency and percentages. The survival time was calculated from the starting date of WBRT until the time of death. Time of death was either recorded from the patient charts or was confirmed telephonically from the relatives of the patient. The KaplanMeier method was used to summarize the overall and median survival times for all groups of patients. The significance of age, sex, KPS before start of WBRT, number of BM, size of BM, presence of other systemic metastases, control of the primary and total dose of WBRT were evaluated in predicting the survival of patients after WBRT with the help of the log-rank test and Cox-proportional hazard model, considering level of significance as $p$ value less than 0.05 .

\section{RESULTS}

Mean ( \pm S.D.) age of patients was $57.0( \pm 11.0)$ years $(30$ to 88 years). Majority of patients $(67.0 \%)$ were of elderly age group (60 years and above). Male to female ratio was 1.37. Most common symptoms reported by patients were sensory-motor (SM) deficits (51.5\%) followed by headache \& vomiting $(32.0 \%)$ and seizures (13.4\%). One-fourth of patients $(26.8 \%)$ also had extra-cranial metastasis. Majority of the patients (70.1\%) had lung as the primary site of tumour, followed by cancer breast (9.3\%). $12.3 \%$ of patients had their primary at 
colon, kidney, base of tongue and other rare sites. Nearly half of patients (46.4\%) had up front brain mets. KPS was 60 and above in $72.2 \%$ of patients before start of WBRT, and in $77.3 \%$ of patients after WBRT. Majority of the patients (84.5\%) had completed radiotherapy. Chemotherapy was received by 37 patients (38.1\%). Out of 97 patients, 10 patients were alive at the date of last contact while four patients were lost to follow up. Median survival was found to be 3.7 months ( 0.03 to 21.9 months). Prognostic factors for survival in patients with brain metastases are shown in Table 1 and Table 2. On univariate analysis, median survival was found to be significantly higher among patients with less than three brain metastasis ( $p=0.003$, Fig. 1), size of brain metastasis less than $3 \mathrm{cms}$ ( $p=0.005$, Fig. 2) and dose of radiotherapy more than or equal to 30 Gy ( $p=0.001$, Fig. 3). Age, gender, KPS at presentation, extra cranial metastasis and control of primary were not found to be statistically significant prognostic factors of overall survival.

On multivariate analysis also, the number of BM, size of BM and dose of radiotherapy were found to be significant predictors of overall survival. Death rate in patients with three or more $\mathrm{BM}$ was found to be 2.2 times more than lesser number of BM ( $p=0.005$, Hazard ratio: 2.293, CI: 1.287-4.085). Similarly, it was 1.9 times among patients with size of BM three cms or more than patients with size of brain mets less than three cms ( $\mathrm{p}=0.016$, Hazard ratio: 1.992, CI: 1.138-3.484). However, death rate was found to be almost one-tenth $(0.12$ times) in patients who received RT dose of more than or equals 30 Gy than those who received less than 30 Gy.

\begin{tabular}{|c|c|c|c|}
\hline Prognostic Factors & Patients $(\mathrm{N}=83)$ & Median Survival (Months) & $\mathbf{p}$ \\
\hline \multicolumn{4}{|c|}{ Age } \\
\hline$\leq 60$ years & 53 & 3.8 & \multirow{2}{*}{0.269} \\
\hline$>60$ years & 30 & 2.9 & \\
\hline \multicolumn{4}{|c|}{ Gender } \\
\hline Male & 49 & 3.7 & \multirow{2}{*}{0.520} \\
\hline Female & 34 & 3.3 & \\
\hline \multicolumn{4}{|c|}{ KPS at Presentation } \\
\hline$<60$ & 24 & 3.0 & \multirow{2}{*}{0.568} \\
\hline$\geq 60$ & 59 & 3.7 & \\
\hline \multicolumn{4}{|c|}{ Number of Brain mets } \\
\hline$<3$ & 27 & 3.8 & \multirow{2}{*}{$0.003^{*}$} \\
\hline$>3$ & 56 & 3.1 & \\
\hline \multicolumn{4}{|c|}{ Size of Brain mets } \\
\hline$<3 \mathrm{cms}$ & 62 & 3.7 & \multirow{2}{*}{$0.005^{*}$} \\
\hline$\geq 3 \mathrm{cms}$ & 21 & 3.0 & \\
\hline \multicolumn{4}{|c|}{ Extra-Cranial mets } \\
\hline Yes & 24 & 2.9 & \multirow{2}{*}{0.810} \\
\hline No & 59 & 3.7 & \\
\hline \multicolumn{4}{|c|}{ Control of Primary Site } \\
\hline Yes & 23 & 4.7 & \multirow{2}{*}{0.176} \\
\hline No & 60 & 2.5 & \\
\hline \multicolumn{4}{|c|}{ RT Dose } \\
\hline$<30 \mathrm{~Gy}$ & 5 & 0.9 & \multirow{2}{*}{$0.001^{*}$} \\
\hline$\geq 30 \mathrm{~Gy}$ & 78 & 3.7 & \\
\hline \multicolumn{4}{|c|}{$\begin{array}{l}\text { Table 1. Prognostic Factors for Survival in Patients with } \\
\text { Brain Metastases (Univariate Analysis) }\end{array}$} \\
\hline *Significant & & & \\
\hline
\end{tabular}

\begin{tabular}{|c|c|c|c|}
\hline Prognostic Factors & $\mathbf{p}$ & Hazard Ratio & 95\% CI* \\
\hline Number of BM & 0.005 & 2.293 & $1.287-4.085$ \\
\hline Size of BM & 0.016 & 1.992 & $1.138-3.484$ \\
\hline RT Dose & 0.000 & 0.129 & $0.046-0.361$ \\
\hline \multicolumn{3}{|c|}{ Table 2. Prognostic Factors for Survival of Patients } \\
(Multivariate Analysis) \\
\hline${ }^{*}$ CI: Confidence interval
\end{tabular}
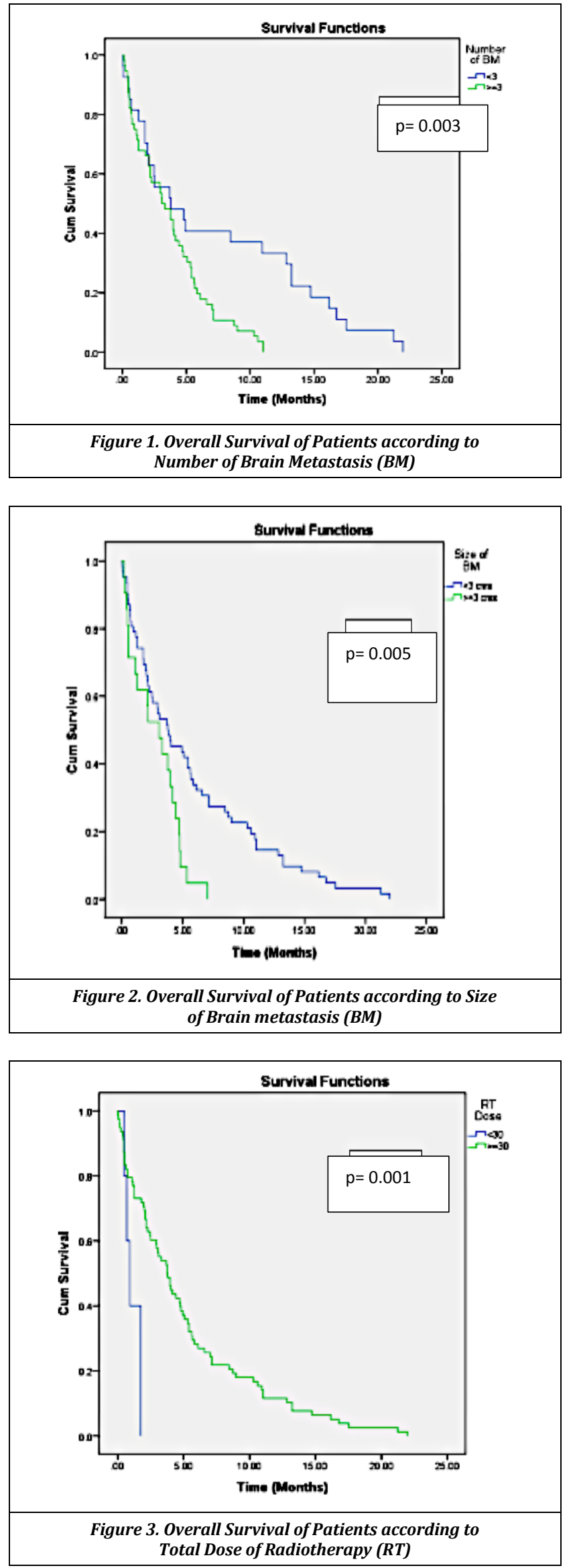
DISCUSSION

Brain metastasis is a dreaded complication affecting many patients with end stage malignancy. These events are a major source of morbidity and can significantly decrease a patient's quality of life. Improvement in survival outcomes in patients with BM still remains a big challenge for the oncologists, despite the advent of stereotactic radiotherapy and novel chemotherapeutic agents available.

Very few patients of brain secondaries are eligible for resection, as at the time of presentation, patient has already developed multiple brain secondaries, or the disease has increased in size. Moreover, the performance status of the patient rapidly declines after BM. Without effective drugs that are able to cross the blood-brain barrier, radiation has become the workhorse of brain metastasis treatment.

WBRT is the best available option if the performance status of patient allows for interventional treatment. In a country like ours, very few centres are equipped with stereotactic radiotherapy equipment and none is available in our State. Therefore, WBRT is the best radiotherapy treatment offered to patients of BM in developing countries including ours. The role of WBRT is mainly palliative. The efficacy of palliative radiotherapy with respect to relief of neurologic complications and improvements in quality of life has been confirmed by many studies.[9,10,11]

However, whole brain radiation can leave patients with persistent fatigue, permanent hair loss, profound memory problems, and other serious cognitive deficits side effects that may worsen with time, raising serious quality of life issues as patients are able to live longer. Being the only institution in the State providing radiotherapy facility, BM represents a significant proportion of the total palliative radiotherapy workload in the RT centre. Considering the above practical problems, it becomes relevant to identify patients on the basis of prognostic factors who may actually benefit from WBRT and who may only require best supportive care. Though WBRT is widely prescribed as a treatment modality, its effectiveness in improving survival has not been studied in our setting in the past.

There is lack of information available on the prognostic factors affecting survival in patients with brain metastases. No such study is available in our region. In our evaluation, the following were found to be associated with a good prognosis: less than three metastatic lesions, size of metastatic lesion less than $3 \mathrm{~cm}$ and dose of RT more than equal to $30 \mathrm{~Gy}$. A retrospective study was done in 162 breast cancer patients in a single institution in Germany, the significant prognostic factors for survival were Karnofsky Index, dose of radiation, solitary metastases and primary tumour size.[12]

Our results compare well to a study by Lagerwaard et al, where overall median survival was 3.4 months, with 6-month, 1-year, and 2-year survival percentages of $36 \%, 12 \%$, and $4 \%$ respectively. Performance status, response to steroid treatment, primary tumour activity, and serum lactate dehydrogenase were found to be independent prognostic factors with the strongest impact on survival, second only to treatment modality. Site of primary tumour, age, and number of brain metastases were also identified as prognostic factors. ${ }^{[13]}$
Non-randomised studies suggest that WBRT increases median survival up to three to six months. [8,9] Median survival of participants with symptomatic brain metastases was approximately one month without treatment and two months with corticosteroid use. In a study of 75 patients treated by WBRT in a university of Finland, median survival was 4 months. Poor performance status at the time of diagnosis of $\mathrm{BM}$ and presence of other extra cranial metastatic sites were related to short survival.[14]

In a cohort of 232 patients of brain metastasis studied by Schouten et al, the frequency of brain metastases was highest in patients with lung carcinoma, followed by patients with renal carcinoma.[15] In our study population also, cancer lung is the most common primary site, followed by cancer breast.

Liu et al in a study population having brain metastasis from breast cancer analysed the overall survival rate and determined prognostic factors affecting outcomes of these patients. The median overall survival rate was 7.3 months. The 1- and 2-year overall survival rates were 37 and 20\%, respectively. In multivariate analysis, KPS and number of brain metastases were significant prognostic factors for overall survival.[16]

Higher dose of WBRT is a significant predictor of survival in our study. There remains a debate as to the optimal fractionation/dose schedule of WBRT for patients with brain metastases. Some believe that a higher dose/ hyper fractionated regimen may improve survival in patients with a favourable prognosis.[17] Evidence indicates that various fractionation schedules are equivalent.

Literature review done by Tsao $\mathrm{MN}$ et al published in Cochrane library revealed that none of the included randomised controlled trials found benefit (In terms of overall survival, neurological function, or symptom control)with higher biological altered dose fractionation schedules as compared with standard doses (3000 cGy in 10 fractions, or 2000 cGy in 4 or 5 daily fractions). Two studies provided data on comparison of two fractionation schedules commonly employed in Canada (2000 cGy in 5 fractions, or 3000 cGy in 10 fractions).[18,19] Overall survival and neurological function were similar between these two fractionation schemes. Higher dose/hyper fractionated regimen may have survival benefit in patients with a favourable prognosis, but evidence suggests that various fractionation schedules are equivalent.[7,11,18] Patients with a life-expectancy of greater than 6 months should receive at least or equal to 2.0 Gy per fraction to minimize the risk of radiation-induced leukoencephalopathy and dementia.[20] The most common dose fractionation schedule in practice is $30 \mathrm{~Gy}$ in 10 fractions over the course of two weeks. The same is followed at our institute in majority of patients. Other dose schedules used at our cancer centre is 20 Gy in 5 fractions and 40 Gy in 20 fractions. The results of present study clearly show significantly better survival in those who received RT dose up to or more than $30 \mathrm{~Gy}$.

Prognostic factors may vary by diagnosis. A multiinstitutional retrospective study on 3940 patients was done to define the diagnosis-specific Graded Prognostic Assessment (GPA) prognostic indices, a prognostic index for patients with brain metastases. For lung cancer, prognostic factors were Karnofsky performance score, age, presence of extra cranial metastases, and number of brain metastases, for melanoma and renal cell cancer, prognostic factors were Karnofsky performance score and the number of brain metastases. For 
breast cancer, prognostic factors were tumour subtype, Karnofsky performance score, and age. For GI cancer, the only prognostic factor was the Karnofsky performance score. The overall median survival time for all patients was 7.16 months, but it varied from 2.79 to 25.30 months depending on diagnosis and GPA.[21]

Apart from recursive partitioning analysis, an alternative prognostic scoring system has been derived from patients undergoing SRS, called the score index for radiosurgery (SIR), which takes into account not only age, KPS score, and systemic disease status but also lesion number and largest lesion volume. [22] Oligometastatic disease can be addressed with surgery apart from radiotherapy and thus, expected to have a better outcome than non resectable disease.

Kim et al evaluated the effectiveness of WBRT followed by stereotactic radiotherapy for newly diagnosed brain metastasis in 25 patients. The overall median survival was 15 months and the actuarial survivals at 1 - and 2-year were $67 \%$ and $31 \%$, respectively. They concluded that cranial radiotherapy followed by stereotactic radiotherapy was useful in the local control of metastatic tumours, and in the survival of patients with tumour factors, such as small size or the absence of extra cranial tumour activity, and host factors, such as young age or good performance status.[23]

\section{Limitations}

There were several limitations of the study. This was a retrospective study with a small sample. Some patients were lost to follow up and data regarding their treatment was not available. RPA class scoring is not done in the present study. The effect of steroids in the improvement of symptoms and quality of life was not taken into consideration.

\section{CONCLUSIONS}

Though WBRT improves survival in patients, the benefit offered is small. Based on prognostic factors, we can identify such group of patients who may just be offered best supportive care. This may decrease the palliative workload in a limited resource setting like ours and also, reduce the hospital stay of the patients and allow them to spend more time with their family members. To the best of our knowledge, this is the first single center study reported at a tertiary care hospital from our region. More studies of similar kind should be conducted to determine the prognostic factors affecting survival in metastatic brain tumour. Also, studies depicting improvement in quality of life after WBRT in our study population may be helpful in deciding interventional treatment.

\section{REFERENCES}

[1] Loeffler JS, Patchell RA, Sawaya R. Treatment of metastatic cancer. In: Devita VT, Hellman S, Rosenberg SA, eds. Cancer: Principles and Practice of Oncology. $5^{\text {th }}$ edn. Philadelphia: Lippincott-Raven Publication 1997: p. 2523.

[2] Lin NU, Winer EP. Brain metastases: the HER2 paradigm. Clin Cancer Res 2007;13(6):1648-55.
[3] Tham YL, Sexton K, Kramer R, et al. Primary breast cancer phenotypes associated with propensity for central nervous system metastases. Cancer 2006;107(4):696704.

[4] Arrieta 0, Villarreal-Garza C, Zamora J, et al. Long-term survival in patients with non-small cell lung cancer and synchronous brain metastasis treated with whole-brain radiotherapy and thoracic chemoradiation. Radiat Oncol 2011;6:166.

[5] Markesbery WR, Brooks WH, Gupta GD, et al. Treatment for patients with cerebral metastases. Arch Neurol 1978;35(11):754-6.

[6] Posner JB. Management of central nervous system metastasis. Semin Oncol 1977;4(1):81-91.

[7] Tsao MN, Lloyd N, Wong RK, et al. Whole brain radiotherapy for the treatment of newly diagnosed multiple brain metastases. Cochrane Database Syst Rev 2012;(4):CD003869.

[8] Katz HR. The relative effectiveness of radiation therapy, corticosteroids and surgery in the management of melanoma metastatic to the central nervous system. International Journal of Radiation Oncology, Biology, Physics 1981;7(7):897-906.

[9] Harwood AR, Simson WJ. Radiation therapy of cerebral metastases: a randomized prospective clinical trial. Int J Radiat Oncol Biol Phys 1977;2(11-12):1091-4.

[10] Gelber RD, Larson M, Borgelt BB, et al. Equivalence of radiation schedules for the palliative treatment of brain metastases in patients with favorable prognosis. Cancer 1981;48(8):1749-53.

[11] Oneschuk D, Bruera E. Palliative management of brain metastases. Support Care Cancer 1998;6(4):365-72.

[12] Lentzsch S, Reichardt P, Weber F, et al. Brain metastases in breast cancer: prognostic factors and management. Eur J Cancer 1999;35(4):580-5.

[13] Lagerwaard FJ, Levendag PC, Nowak PJ, et al. Identification of prognostic factors in patients with brain metastases: a review of 1292 patients. Int J Radiat Oncol Biol Phys 1999;43(4):795-803.

[14] Sundstrom JT, Minn H, Lertola KK, et al. Prognosis of patients treated for intracranial metastases with wholebrain irradiation. Ann Med 1998;30(3):296-9.

[15] Schouten LJ, Rutten J, Huveneers HA, et al. Incidence of brain metastases in a cohort of patients with carcinoma of the breast, colon, kidney and lung and melanoma. Cancer 2002;94(10):2698-705.

[16] Liu MT, Hsieh CY, Wang AY, et al. Prognostic factors affecting the outcome of brain metastasis from breast cancer. Support Care Cancer 2006;14(9):936-42.

[17] Khuntia D, Brown P, Li J, et al. Whole-brain radiotherapy in the management of brain metastasis. J Clin Oncol 2006;24(8):1295-304.

[18] Chatani M, Matayoshi Y, Masaki N, et al. Radiation therapy for brain metastases from lung carcinoma. Prospective randomized trial according to level of lactate dehydrogenase. Strahlenther Onkologie 1994;170(3):155-61.

[19] Borgelt B, Gelber R, Kramer S, et al. The palliation of brain metastases: final results of the first two studies by the Radiation Therapy Oncology Group. International Journal of Radiation Oncology, Biology, Physics 1980;6(1):1-9. 
[20] Sneed PK, Larson DA, Wara WM. Radiotherapy for cerebral metastases. Neurosurgery Clinics of North America 1996;7(3):505-16.

[21] Sperduto PW, Kased N, Roberge D, et al. Summary report on the graded prognostic assessment: an accurate and facile diagnosis-specific tool to estimate survival for patients with brain metastases. J Clin Oncol 2012;30(4):419-25.
[22] Eichler AF, Loeffler JS. Multidisciplinary management of brain metastases. The Oncologist 2007;12(7):884-98.

[23] Kim HJ, Hong S, Kim S, et al. treatment outcome of brain metastasis after the cranial radiotherapy followed by fractionated stereotactic radiotherapy and its prognostic factors. Cancer Research and Treatment 2002;34(4):2848. 